\title{
Modified rehabilitation exercises for mild cases of COVID-19
}

\author{
Lulu Zha ${ }^{1,2}$, Xi Xu ${ }^{1,2}$, Dongya Wang ${ }^{1,2}$, Guibin Qiao ${ }^{3,4}$, Weitao Zhuang ${ }^{3,4}$, Shujie Huang ${ }^{3,4} \wedge$ \\ ${ }^{1}$ General Hospital of Southern Theater Command, Chinese People's Liberation Army, Guangzhou, China; ${ }^{2}$ Huoshenshan Hospital, Wuhan, China; \\ ${ }^{3}$ Department of Thoracic Surgery, Guangdong Provincial People's Hospital, Guangdong Academy of Medical Sciences, Guangzhou, China; ${ }^{4}$ Shantou \\ University Medical College, Shantou, China \\ Contributions: (I) Conception and design: G Qiao; (II) Administrative support: G Qiao; (III) Provision of study materials or patients: L Zha, D Wang; \\ (IV) Collection and assembly of data: S Huang, W Zhuang; (V) Data analysis and interpretation: S Huang; (VI) Manuscript writing: All authors; (VII) \\ Final approval of manuscript: All authors. \\ Correspondence to: Shujie Huang, MBBS; Weitao Zhuang, MBBS. Department of Thoracic Surgery, Guangdong Provincial People's Hospital, \\ Guangdong Academy of Medical Sciences, 106 Zhongshan Second Road, Guangzhou, China. Email: 15sjhuang2@stu.edu.cn; wtzhuang1@163.com.
}

Background: Coronavirus disease 2019 (COVID-19) caused by a new Betacoronavirus severe acute respiratory syndrome coronavirus 2 (SARS-CoV-2) is currently a global pandemic. Gathered clinicopathological evidence in COVID-19 patients shows that alveoli injuries and interstitial changes are the major mechanisms of impaired O2/CO2 exchange. Few rehabilitation exercises concerning COVID-19 patients were reported. Here, we present a modified version of rehabilitation exercises based on the underlying mechanism of the disease to mild cases of COVID-19. These exercises aimed to improve the pulmonary function of patients and ease the expectoration process. Additionally, an essential branch of Traditional Chinese Medicine (TCM) named acupressure was integrated into the exercises to facilitate the recovery and maintenance of pulmonary function.

Methods: From March 4, 2020 to May 5, 2020, a total of 60 COVID-19 patients who completed the full course of MRE were enrolled in this observational study. The diagnostic and classification criteria were based on the $7^{\text {th }}$ edition of Diagnosis and Treatment Guideline of COVID-19 published by the National Health Commission of the People's Republic of China. We prospectively gathered patients' reported outcomes concerning respiration-related symptoms at four different time points, including: (I) at admission; (II) at the time of hospital discharge; (III) two weeks after discharge; (IV) four weeks after discharge. The reported respiratory symptoms included dry cough, productive cough, difficulty in expectoration, and dyspnea.

Results: In total, 60 confirmed mild COVID-19 cases were enrolled with a median age of 54 years old. The baseline prevalence for dry cough, productive cough, difficulty in expectoration, and dyspnea were $41.7 \%$, $43.3 \%, 35.0 \%$, and $50.0 \%$, respectively. The pronounced decline in symptom prevalence was recorded over time. Interestingly, four weeks after discharge, we noticed a lower remission rate in productive cough and difficulty in expectoration.

Conclusions: The modified rehabilitation exercises were retrieved from the Eight-Section Brocade, and are specifically designed for rehabilitation of COVID-19 patients at home or health facilities. Based on current findings on pronouncedly improved remission rate in respiratory symptoms, we recommend the MRE as suitable rehabilitation exercise to smooth respiration and ease the expectoration process in mild COVID-19 cases.

Keywords: Coronavirus disease 2019 (COVID-19); severe acute respiratory syndrome coronavirus 2 (SARS-CoV-2); rehabilitation; exercise therapy

Submitted Mar 19, 2020. Accepted for publication Jul 16, 2020.

doi: $10.21037 / \mathrm{apm}-20-753$

View this article at: http://dx.doi.org/10.21037/apm-20-753

^ORCID: Weitao Zhuang, 0000-0002-8679-4109; Shujie Huang, 0000-0002-2339-0684. 


\section{Introduction}

Coronavirus disease 2019 (COVID-19) is an infectious disease caused by a new Betacoronavirus severe acute respiratory syndrome coronavirus 2 (SARS-CoV-2). In early December 2019, it began to draw public's attention. Over time, COVID-19 has increasingly been recognized as an important global public health concern. On January 30, 2020, the World Health Organization (WHO) declared the outbreak a public health emergency of international concern (PHEIC) (1). Later, on March 11, 2020, the WHO named that COVID-19 can be characterized as a pandemic (2) after assessing the infection rates. According to the WHO, the flare of COVID-19 has spread to 216 countries, areas or territories with more than $7,800,000$ confirmed cases and 431,541 deaths (3).

Clinical pictures of COVID-19 may vary from slight discomfort to more severe consequences such as dyspnea, hypoxia, respiratory failure and the worst of all, death (4). In a report released from the Chinese Center for Disease Control and Prevention, approximately 44,500 confirmed cases were classified as mild (81\%), severe (14\%) and critical (5\%) based on disease severity (4).

Despite the little information on the pathogenesis of COVID-19, complementary pathological and radiographic findings have revealed the characteristics of SARS-CoV-2 infection. An autopsy report of a 50-year-old male patient showed bilateral diffuse alveolar damages with cellular fibromyxoid exudates, along with characteristics of acute respiratory distress syndrome (ARDS) such as pneumocytes desquamation, hyaline membrane formation, and interstitial mononuclear inflammatory infiltrates (5). Radiological findings of COVID-19 include ground grass opacity, interlobular septal thickening and patchy consolidation (6-8).

The current management strategy for COVID-19 is mainly supportive care, whereas specific anti-virus medications and vaccines are still being developed. Based on the current laboratory and clinical evidence, it is clear that the key factors causing COVID-19 patients' pulmonary function abnormalities (e.g., $\mathrm{O}_{2} / \mathrm{CO}_{2}$ exchange impairment, ventilation difficulty) are alveolar injuries and interstitial changes, which brings up the discussion of specified treatment and rehabilitation care in accordance with the underlying pathogenesis of the disease process.

Most patients, particularly those diagnosed with mild cases can recover from the disease. However, during the period of rehabilitation, preexisting respiratory symptoms such as dry cough, sputum production can progress for some time. This can result in decreased quality of life. For certain groups of the population, mainly the elderly and patients with preexisting respiratory disorders (e.g., COPD, restrictive lung diseases), it is difficult to expectorate smoothly due to weakened respiratory muscles. Currently, various exercises are introduced to help tackle the above problems along disease recovery process (9-13). However, most of them focus only on the training of respiratory muscles, without paying attention to the specific pathological alterations of COVID-19. In this regard, we designed a modified version of rehabilitation exercises, aiming to smooth respiration and ease the process of expectoration. We present the following article in accordance with the STROBE reporting checklist (available at http://dx.doi.org/10.21037/apm-20-753).

\section{Methods}

\section{Study participants}

From March 4, 2020 to May 5, 2020, 60 patients with mild COVID-19 from Huoshenshan Hospital were voluntary to complete the full course of MRE. The diagnostic criteria were based on the $7^{\text {th }}$ edition of Diagnosis and Treatment Guideline of COVID-19 published by the National Health Commission of the People's Republic of China (14). Patients presented with mild symptoms and no CT evidence of pneumonia on admission were listed as mild cases after confirmation of positive real-time polymerase chain reaction (RT-PCR) results, sequencing of highly homologous viral gene regions, or positive SARS-CoV-2 pathogen antibody testing. Patients who could not complete the full course of MRE or refuse to report their symptom severity over time were excluded.

\section{Ethical statement}

The current study was approved by the Huoshenshan Hospital ethics committee (No. IEC-AF-SL-02) and inform consent was obtained from each participant who had been recruited. The study conformed to the provisions of the Declaration of Helsinki (as revised in 2013).

\section{Training techniques}

The modified rehabilitation exercise (MRE), retrieved from Chinese martial art Eight-section Brocade, is a full-body exercise designed to reduce total airway resistance, smooth 

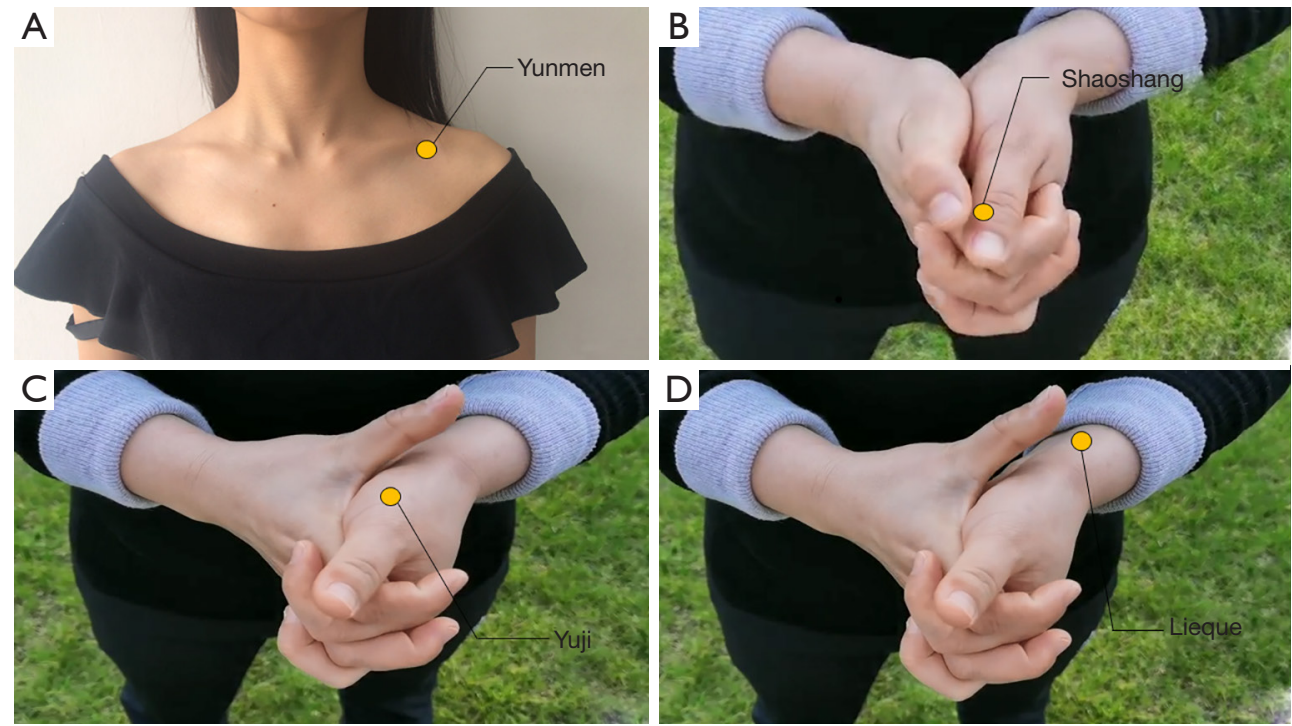

Figure 1 Locations of essential acupoints. (A) Yunmen, located in the infraclavicular fossa, 6 cun lateral to the anterior median line; (B) Shaoshang, located 0.1 cun posterior to the thumb nail (radial side); (C) Yuji, located on the midpoint of the $1^{\text {st }}$ metacarpal (radial side); (D) Lieque, located in the fossa superior to the styloid process of the radius. Detailed technical aspects are described in the text. One cun is equal to the length between the distal interphalangeal joint and the proximal interphalangeal joint on the middle finger.

fresh airflow and improve $\mathrm{O}_{2} / \mathrm{CO}_{2}$ exchange efficiency. MRE can be divided into four sets: Overhead Chest and Shoulder Stretch ( $1^{\text {st }}$ set), Standing Heel Raises and Upper Body Acupressure ( $2^{\text {nd }}$ set), Upper Body Rotation ( $3^{\text {rd }}$ set $)$ and Hand Acupressure Massage ( $4^{\text {th }}$ set). Detailed training techniques for each set of exercises are provided below. A video demonstration of the MRE was also available (Video 1).

\section{Set 1: overhead chest and shoulder stretch (1 set of 2 repetitions)}

Starting off deep inspiration to expand the chest fully while stretching the chest and shoulder in an upright position to maximally open up alveolar sacs, providing sufficient space for $\mathrm{O}_{2} / \mathrm{CO}_{2}$ exchange. The emphasis here is to hold a breath at the end of each inspiration. Besides one can narrow the space between lips during expiration to create certain airway resistance, thus preventing potential airway collapse in some patients.

Set 2: standing heel raises and upper body acupressure ( 2 sets of 12 repetition)

The $2^{\text {nd }}$ set of MRE is standing heel raises while applying upper body acupressure by patting the Yunmen acupoint (Figure 1). The rationale for this is that constant up-anddown body movements through heel raise aid in loosening the adherence between mucus and the epithelial lining of the respiratory tract. Additionally, from the perspective of Traditional Chinese medicine (TCM), applying pressure on lung-associated acupoints will smooth the breathing activity as well as the expectoration process.

\section{Set 3: upper body rotation (1 set of 4 repetitions)}

The $3^{\text {rd }}$ set is upper body rotation while patting the lateral side of the thoracic cavity. The angular momentum initiated by the rotating motion of the thorax plus the vibration produced by patting will further ease the process of mucus clearance.

\section{Set 4: hand acupressure massage ( 3 sets of 12 repetitions)}

The last set of MRE is to massage the three lung-associated acupoints on each hand. In consideration of finding a balance between the physical load of the exercises and the limited physical capacity of patients. We designed the last set to be simple hand exercise which only requires the patients to massage three lung meridian acupoints named Shaoshang, Yuji and Lieque (Figure 1), aiming to facilitate the recovery of patients' respiratory function and combat fatigue.

The full course of MRE is 6-8 repetitions. We suggest 
Table 1 Over-time prevalence of self-reported respiratory symptoms in mild COVID-19 cases

\begin{tabular}{|c|c|c|c|c|}
\hline Self-reported respiratory symptoms & \multicolumn{4}{|c|}{ Time point $\mathrm{n}(\%)$} \\
\hline \multicolumn{5}{|l|}{ Dry cough } \\
\hline Yes & $25(41.7)$ & $10(16.7)$ & $9(15.0)$ & $7(11.7)$ \\
\hline No & $35(58.3)$ & $50(83.3)$ & $51(85.0)$ & $53(88.3)$ \\
\hline Yes & $26(43.3)$ & $12(20.0)$ & $6(10.0)$ & $7(11.7)$ \\
\hline No & $34(56.7)$ & $48(80.0)$ & 54 (90.0.) & $53(88.3)$ \\
\hline \multicolumn{5}{|l|}{ Difficulty in expectoration } \\
\hline Yes & $21(35.0)$ & $4(6.7)$ & $3(5.0)$ & $5(8.3)$ \\
\hline Yes & $30(50.0)$ & $13(21.7)$ & $12(20.0)$ & $9(15.0)$ \\
\hline No & $30(50.0)$ & $47(78.3)$ & $48(80.0)$ & $51(85.0)$ \\
\hline
\end{tabular}

COVID-19, coronavirus disease 2019.

patients to do these exercises at least twice a day.

\section{Study design}

We prospectively collected patient's reported outcomes regarding the severity of their presented respiratory symptoms on four different time points: first at admission, second at the time of discharge, then two and four weeks after they were discharged. The reported respiratory symptoms included dry cough, productive cough, difficulty in expectoration and dyspnea.

\section{Statistical analysis}

The remission rate of each respiratory symptom was calculated via the frequency module of SPSS version 26 (SPSS, Inc, Chicago, Illinois, USA). The trend of remission rates was depicted using GraphPad Prism 8.0 (GraphPad Software, La Jolla, CA, USA).

\section{Results}

\section{Clinical information of the study population}

Overall, 60 confirmed mild COVID-19 cases were enrolled for analyses. Among them, 39 (65\%) were male, and the median age was 54 with an interquartile range (IQR) of 38-62.

\section{Self-reported severity of respiratory symptoms development}

Descriptions of the investigated respiratory symptom prevalence at four different time points were presented in Table 1. The baseline prevalence for dry cough, productive cough, difficulty in expectoration and dyspnea were $41.7 \%$, $43.3 \%, 35.0 \%$ and $50.0 \%$, respectively, which all decreased pronouncedly over time. After one month, the prevalence rate was $11.7 \%$ in dry cough, $11.7 \%$ in productive cough, $8.3 \%$ in difficulty in expectoration, and only $15 \%$ of patients reported dyspnea as a remained symptom. According to the depicted remission rate of these clinical manifestations at four different time points (Figure 2), patients with mild cases who performed MRE had a higher remission rate compared to the baseline values. It was worth mentioning that refractory symptoms still persisted in some patients, which contributed to a lower remission rate in productive cough and difficulty in expectoration four weeks after discharge.

\section{Discussion}

In the past few decades, various rehabilitation therapies have been applied to facilitate the recovery process of patients with respiratory diseases. van der Lee et al. reported after-hours respiratory physiotherapy for patients with community acquired pneumonia (CAP) in 


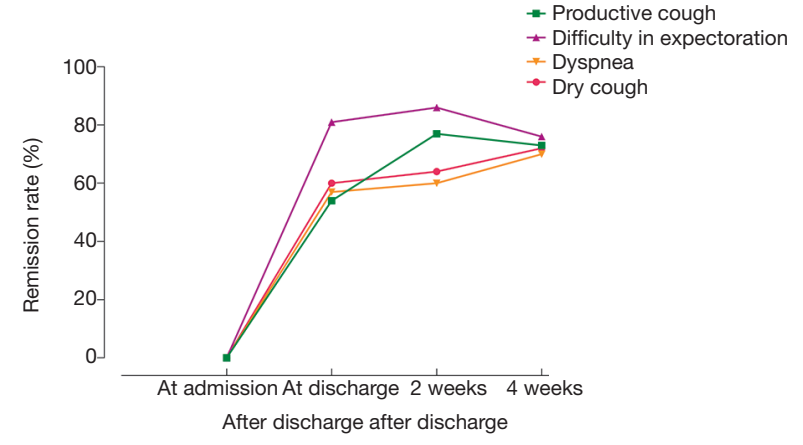

Figure 2 Over-time alterations in respiratory symptom remission rates. Pronounced improvement in respiration-related symptoms in mild COVID-19 patients who performed the full course of MRE during the follow-up period. The maximum slope for the remission rate of each symptom occurred during the hospitalization period. Among the four investigated symptoms, the remission rate of "productive cough" and "difficulty in expectoration" dropped one month after hospital discharge, while remission rates of "dyspnea" and "dry cough" continue to increase. COVID-19, coronavirus disease 2019.

Australia (9). Elsewhere, a study evaluated a communitybased pulmonary rehabilitation involving upper and lower limb duration training on patients suffered from chronic obstructive pulmonary disease (COPD), the results of which were characterized to be safe, feasible and effective (10). A randomized controlled trial (RCT) of integrated physical therapy focusing on pulmonary function training and active upper limb exercise carried out by López-López et al. showed improved physical and functional performance in elderly patients with pneumonia (11). Although different rehabilitation therapies seemed to show positive effects on patients with various respiratory diseases, an RCT study carried out by Greening et al. indicated early rehabilitation intervention in patients with acute exacerbation of chronic obstructive pulmonary disease (AECOPD) did not reduce the risk of hospital readmission rate but increased mortality (12).

The vast majority of pulmonary rehabilitation exercises mainly focused on improving pulmonary function through muscle function training $(10,11,13)$, and the selected subjects were mostly COPD patients $(10,12,13)$. Exercises designed for COPD patients mainly shed light on improving airflow limitation due to irreversible airway and alveolar alterations. Expiratory muscle function training was emphasized in such studies to ensure $\mathrm{CO}_{2}$ exhalation. Distinct pathological differences hinder the applications of these exercises on the rehabilitation of COVID-19 patients. Till now, little attention has been payed to the design of rehabilitation exercises based on the underlying pathogenesis of the disease. Moreover, with the increasing number of COVID-19 patients worldwide, urgent actions are required to design suitable rehabilitation exercises to enhance the patient recovery process. In this regard, we proposed the MRE to COVID-19 patients.

The current study found that pronounced improvement occurred in all four investigated respiratory symptoms in COVID-19 patients who performed the MRE during both hospitalization and quarantine period. The improved rates were the fastest during hospitalization, which remained relatively constant during the next study time point. However, relapsed symptoms such as productive cough and difficulty in expectoration were observed in the fourth time point.

We recommend several perspectives based on the current findings. First, the declined remission rate indicated that the MRE may relieve respiration-related symptoms. The primary purpose of performing the MRE is to smooth the expectoration via sequential steps involved in the exercises. Surprisingly, patients who completed the full course of the MRE also experienced relieved symptoms of dyspnea and dry cough. Second, though a higher recovery rate was noticed four weeks after discharge compared to baseline prevalence, lower remission rates were also observed. During the follow-up process, some patients reported that they had a brief pause of performing MRE 2 weeks after discharge due to multiple reasons. The exact time and duration of pausing the MRE could not be recalled precisely. Thus, no scientific data could be transformed and analyzed. We suspected that pausing the MRE could be attributed to the reduction of remission rates.

There are two distinguishing features of MRE, that is, essential theories of TCM on pulmonary function maintenance are integrated into the exercises. Second, the respiratory exercise in MRE, is accordingly designed to fit the underlying mechanism of respiratory function impairment caused by SARS-CoV-2 infection.

The MRE is originated from ancient Chinese martial art the Eight-Section Brocade. It has been suggested to play an important role in human endocrine (15), CNS, and cardiopulmonary systems (16). Additionally, acupressure, a form of massage therapy utilizing pressure with thumbs or fingertips to stimulate discrete points on the body for relief of a variety of symptoms, is integrated into MRE for rehabilitation of mild COVID-19 cases (17). From the 
perspective of TCM, stimulating certain acupoints through acupressure relieves the respiratory symptoms and improve pulmonary functions. In the $2^{\text {nd }}$ and $4^{\text {th }}$ sets of MRE, lungassociated acupoints (Yunmen, Lieque, Yuji and Shaoshang) acupressure massage (Figure 1) helped to smooth breathing activities and ease the expectoration process.

To date, research regarding the pathophysiology of COVID-19 is still limited. However, gathered evidence based on laboratory findings and clinicopathological characteristics of COVID-19 patients suggest that a large amount of mucus secretion in the airway impedes the process of ventilation and alveoli injuries, together with interstitial changes are underlying mechanisms of impairment of gas exchange. Consequently, we targeted reversible pulmonary alterations such as mucus impediment and interstitial liquid accumulation, and designed specific sets of an exercise to maximize the space of alveolar sacs (set 1) as well as to speed up the mucus clearance process (set 2 and set 3 ).

A previous study suggested that early rehabilitation exercise in patients with the exacerbated disease is associated with a higher mortality rate (12). Therefore, we are cautious about both the timing of exercise implementation and selection of patient population according to disease severity. Considering the balance between physical load and physical capacity of the patients, we hereby recommend patients who are characterized as mild COVID-19 cases to carry out the exercises daily.

Currently, MRE is well received by patients in Wuhan due to its simplicity and potential benefit for nearly all age groups. However, MRE is only implemented in a small group of patients in Huoshenshan Hospital. Further studies are required to validate the effectiveness of this rehabilitation exercise.

In summary, the modified rehabilitation exercises are specifically designed for rehabilitation of COVID-19 patients at home or health facilities. Currently, it is only recommended for the mild COVID-19 patients. Incorporated with well-designed movements that based on TCM theories and specific mechanisms of SARS$\mathrm{CoV}-2$ infection, MRE is hereby considered as suitable rehabilitation exercise to smooth respiration and ease the expectoration process.

\section{Acknowledgments}

We thank the nurse staff and the health workers who contributed to the field work. We are grateful to the Freescience Editing Service for English language editing. Funding: None.

\section{Footnote}

Reporting Checklist: The authors have completed the STROBE reporting checklist. Available at http://dx.doi. org/10.21037/apm-20-753

Data Sharing Statement: Available at http://dx.doi. org/10.21037/apm-20-753

Conflicts of Interest: All authors have completed the ICMJE uniform disclosure form (available at http://dx.doi. org/10.21037/apm-20-753). The authors have no conflicts of interest to declare.

Ethical Statement: The authors are accountable for all aspects of the work in ensuring that questions related to the accuracy or integrity of any part of the work are appropriately investigated and resolved. The current study has been approved by the Huoshenshan Hospital ethics committee (No. IEC-AF-SL-02) and inform consent was obtained from each participant who had been recruited. The study conformed to the provisions of the Declaration of Helsinki (as revised in 2013).

Open Access Statement: This is an Open Access article distributed in accordance with the Creative Commons Attribution-NonCommercial-NoDerivs 4.0 International License (CC BY-NC-ND 4.0), which permits the noncommercial replication and distribution of the article with the strict proviso that no changes or edits are made and the original work is properly cited (including links to both the formal publication through the relevant DOI and the license). See: https://creativecommons.org/licenses/by-nc-nd/4.0/.

\section{References}

1. World Health Organization. Novel coronavirus situation report-11. 2020. Available online: https://www. who.int/docs/default-source/coronaviruse/situationreports/20200131-sitrep-11-ncov.pdf?sfvrsn=de7c0f7_4. Accessed March 132020.

2. World Health Organization. Novel coronavirus situation report-51.2002. Available online: https:// 
www.who.int/docs/default-source/coronaviruse/ situation-reports/20200311-sitrep-51-covid-19. pdf? sfvrsn=1ba62e57_10. Accessed March 132020.

3. World Health Organization. Coronavirus disease (COVID-19) outbreak situation. 2020. Available online: https://www.who.int/emergencies/diseases/novelcoronavirus-2019. Accessed June 16, 2020.

4. Wu Z, McGoogan JM. Characteristics of and Important Lessons From the Coronavirus Disease 2019 (COVID-19) Outbreak in China: Summary of a Report of 72314 Cases From the Chinese Center for Disease Control and Prevention. JAMA 2020;323:1239-42.

5. Xu Z, Shi L, Wang Y, et al. Pathological findings of COVID-19 associated with acute respiratory distress syndrome. Lancet Respir Med 2020;8:420-2.

6. Pan Y, Guan H, Zhou S, et al. Initial CT findings and temporal changes in patients with the novel coronavirus pneumonia (2019-nCoV): a study of 63 patients in Wuhan, China. Eur Radiol 2020;30:3306-9.

7. Zhao W, Zhong Z, Xie X, et al. Relation Between Chest CT Findings and Clinical Conditions of Coronavirus Disease (COVID-19) Pneumonia: A Multicenter Study. AJR Am J Roentgenol 2020;214:1072-7.

8. Zhu Y, Liu YL, Li ZP, et al. Clinical and CT imaging features of 2019 novel coronavirus disease (COVID-19). J Infect 2020. [Epub ahead of print].

9. van der Lee L, Hill AM, Patman S. After-hours respiratory physiotherapy for intubated and mechanically ventilated patients with community-acquired pneumonia: An Australian perspective. Aust Crit Care 2018;31:349-54.

10. Cecins N, Landers H, Jenkins S. Community-based pulmonary rehabilitation in a non-healthcare facility is feasible and effective. Chron Respir Dis 2017;14:3-10.

11. López-López L, Torres-Sanchez I, Rodriguez-Torres

Cite this article as: Zha L, Xu X, Wang D, Qiao G, Zhuang W, Huang S. Modified rehabilitation exercises for mild cases of COVID-19. Ann Palliat Med 2020;9(5):3100-3106. doi: 10.21037/ apm-20-753
J, et al. Does adding an integrated physical therapy and neuromuscular electrical stimulation therapy to standard rehabilitation improve functional outcome in elderly patients with pneumonia? A randomised controlled trial. Clin Rehabil 2019;33:1757-66.

12. Greening NJ, Williams JEA, Hussain SF, et al. An early rehabilitation intervention to enhance recovery during hospital admission for an exacerbation of chronic respiratory disease: randomised controlled trial. BMJ 2014;349:g4315.

13. Klijn P, Van Keimpema A, Legemaat M, et al. Nonlinear Exercise Training in Advanced Chronic Obstructive Pulmonary Disease Is Superior to Traditional Exercise Training. A Randomized Trial. Am J Respir Crit Care Med 2013;188:193-200.

14. National Health Commission of the People's Republic of China. 7th edition of Diagnosis and Treatment Guideline of COVID-19. 2020. Available online: http://www.nhc. gov.cn/yzygj/s7653p/202003/46c9294a7dfe4cef80dc7f591 2eb1989.shtml. Accessed June 052020.

15. Wang F, Wang W, Zhang R, et al. Clinical observation on physiological and psychological effects of Eight-Section Brocade on type 2 diabetic patients. Journal of Traditional Chinese Medicine 2008;28:101-5.

16. Lv W, Wang X, Liu J, et al. Eight-Section Brocade Exercises Improve the Sleep Quality and Memory Consolidation and Cardiopulmonary Function of Older Adults With Atrial Fibrillation-Associated Stroke. Front Psychol 2019;10:2348.

17. Khanghah AG, Rizi MS, Nabi BN, et al. Effects of Acupressure on Fatigue in Patients with Cancer Who Underwent Chemotherapy. J Acupunct Meridian Stud 2019;12:103-10. 